\section{Zokl2, Poliklinik für heroingestützte Behandlung der ARUD Zürich}

Die ARUD Zürich, Arbeitsgemeinschaft für risikoarmen Umgang mit Drogen, ist ein privatrechtlicher Verein, der sich für die Medizinalisierung des Umgangs mit Drogen einsetzt, die dem Betäubungsmittelgesetz unterstellt sind. Weitere Schwerpunkte sind Evaluations- und Forschungsprojekte im Drogenbereich, Öffentlichkeitsarbeit, politisches Engagement sowie Weiterbildung und Vernetzung von Fachleuten.

Die ARUD betreibt in Zürich zwei Polikliniken:

- Zokl1, Poliklinik für methadongestützte Behandlung mit ca. 500 Plätzen

- Zokl2, Poliklinik für heroingestützte Behandlung mit 120 Plätzen

\section{Zielsetzung der heroingestützten Behandlung im Zokl2}

Grundsätzlich werden bei opiatabhängigen Patient(inn)en drei Ebenen von Behandlungszielen definiert, die möglichst umfassend und oft parallel angegangen werden, aber auch sinnvoll einzeln festgelegt werden können.

\section{Verminderung von Schaden und Leiden (harm reduction)} Die Reduktion von Schaden bezieht sich auf die durch die Illegalität der Drogen erzeugten selbstschädigenden und die Gesellschaft beeinträchtigenden Mechanismen. Dazu gehört auch die Prävention von Infektionserkrankungen, Abszessen bzw. deren medizinische Behandlung bei bereits erfolgter Erkrankung, Verhindern von Überdosierung und Verwahrlosung.

2. Erhalten und Stabilisieren von gesundheitlichen und integrativen Ressourcen

Ein Verlust von bereits erlangten Fähigkeiten soll verhindert werden, um dem Prozess der Marginalisierung entgegenzuwirken.

\section{Ausbau von Ressourcen und psychosoziale Rehabilita-} tion

Durch den Ausbau der psychischen, somatischen und sozialen Fähigkeiten und Fertigkeiten sollen die Autonomie der Patient(inn)en gefördert sowie die bisherige Entwicklung prägende Themen bearbeitet werden, mit dem Ziel der Stärkung der Persönlichkeit.

Abstinenzbemühungen werden aktiv unterstützt, wenn sich dadurch Lebensqualität verbessert.

Suchttherapie 2000; $1: 89-90$

(c) Georg Thieme Verlag Stuttgart · New York

ISSN 1439-9903
Daniel Meili

ARUD Zürich, Poliklinik ZokI2

\section{Geschlechtergerechtes Angebot}

Das Zokl2 legt den Schwerpunkt des therapeutischen Angebots auf geschlechtergerechte Arbeit, d.h., es wird für beide Geschlechter eine adäquate, ihrer Situation möglichst gerecht werdende Behandlung zur Verfügung gestellt. Dies bedingt unter anderem, dass der Frauenanteil aktiv hoch gehalten wird (z.Z. zwei Drittel Frauen, ein Drittel Männer). Therapeutische Gruppen für Frauen, Mütter, Männer und ein Kinderhütedienst sind Elemente dieses Konzepts.

\section{Aufnahmebedingungen}

- mindestens 18 Jahre alt

- seit mindestens zwei Jahren schwer heroinabhängig

- mindestens zwei gescheiterte Behandlungsversuche

- Defizite im somatischen, psychischen oder sozialen Bereich

\section{Psychiatrische und psychosoziale Betreuung}

Mitarbeiter/-innen: $80 \%$ psychiatrischer Oberarzt; therapeutische Mitarbeiter/-innen: 270\% psychosozial/-therapeutisch, $40 \%$ ärztlich. Jeder Patient/jede Patientin wird von Anfang an konstant von einem/einer therapeutischen Mitarbeiter/-in begleitet. Die kontinuierliche Betreuung erfolgt in der Regel im Rahmen von Gruppengesprächen. Je nach Indikation werden spezielle Behandlungsformen gewählt (z.B. Psychotherapie im Einzelsetting, Psychopharmaka-Behandlung, sozialarbeiterische Unterstützung).

\section{Somatische Betreuung}

Mitarbeiter/-innen: 20\% somatischer Oberarzt; 90\% Assistenzärzte/-ärztinnen. Das Zokl2 bietet eine basisorientierte hausärztliche Grundversorgung mit regelmäßiger gynäkologischer Betreuung an. Im infektiologischen Bereich werden die neusten Behandlungen, meistens im Rahmen von Forschungsprojekten, für HIV- und Hepatitis-C-Infektionen angeboten.

\section{Substitution}

Mitarbeiter/-innen für die Abgabe: 450\%. Zur Zeit steht Heroin zur intravenösen Applikation und in zwei Tablettenformen (rasch wirksam und retard) zur Verfügung. Schluckbares Methadon und in einzelnen Fällen Morphium können als Basissubstitution eingesetzt werden. Die rauchbare Form von Heroin, welche in Zigarettenform auf Waldmeisterbasis entwickelt worden war (Tabak darf in der Schweiz medizinisch nicht verordnet werden), bewährte sich nicht und Folienrauchen nach holländischem Muster ist zur Zeit nicht zugelassen. 


\begin{tabular}{|c|c|c|c|}
\hline \multicolumn{2}{|l|}{ Alter } & \multicolumn{2}{|l|}{ Dauer der Abhängigkeit } \\
\hline Alter bis $25 \mathrm{~J} .:$ & $25 \%$ & bis 4 Jahre: & $12 \%$ \\
\hline Alter 26-30: & $35 \%$ & 5-9 Jahre & $45 \%$ \\
\hline Alter 31-35: & $25 \%$ & 10-15 Jahre & $25 \%$ \\
\hline Alter über 35: & $15 \%$ & mehr als 15 Jahre & $15 \%$ \\
\hline Arbeitssituation & & zusätzlicher Drogenkons & \\
\hline Vollzeit/Teilzeit/temporär & $23 \%$ & Kokain & $75 \%$ \\
\hline arbeitslos & $30 \%$ & Benzodiazepine & $40 \%$ \\
\hline Rente & $17 \%$ & & \\
\hline nicht auf Arbeitssuche/delinquent & $30 \%$ & & \\
\hline $\begin{array}{l}\text { Schulden zwischen } \\
\text { Fr. 5000,- und Fr. } 30000,-\end{array}$ & $70 \%$ & HIV-Prävalenz & $23 \%$ \\
\hline Sozialhilfeempfänger & $53 \%$ & & \\
\hline $\begin{array}{l}\text { hauptsächlich illegales Einkommen/ } \\
\text { Grauzone }\end{array}$ & $57 \%$ & $\begin{array}{l}\text { Wechsel aus unstabiler } \\
\text { Methadonbehandlung }\end{array}$ & $50 \%$ \\
\hline
\end{tabular}

Tab.1 Patient(inn)encharakteristika bei Eintritt

Stichproben über drei Zeitabschnitte n $=141$, $80 \%$ Frauen, 20\% Männer

\section{Kosten}

Pro Behandlungsplatz kosten die Schweizer Heroinprojekte zwischen CHF 55,- und 80,-, je nach Größe (Patientenzahl) und Behandlungsintensität (Personal-/Patientenschlüssel).

\section{Erfahrungen}

Haltequote im Zokl2 über 6 Monate: $80 \%$

- von 76 Eintritten von Januar 1994 bis März 1995: 30 Patient(inn)en im September 1999 noch im Programm

- von 55 Eintritten von April 1995 bis März 1996: 28 Patient(inn)en im September 1999 noch im Programm

Tab.2 Austrittsgründe

\begin{tabular}{ll}
\hline abstinenzorientierte Therapie & $20 \%$ \\
methadongestützte Behandlung & $41 \%$ \\
Abbruch & $26 \%$ \\
andere Gründe & $13 \%$ \\
\hline
\end{tabular}

Generell verbesserte sich bei einem beträchtlichen Teil der Patient(inn)en der psychische Zustand (v.a. Verminderung von depressiven Störungen und Angstsymptomen) und die somatische Situation (Verbesserung des Allgemeinzustands, Abnahme von Hautinfektionen etc.). Die Verbesserung der sozialen Reintegration lässt sich vor allem an einer eindrücklichen Reduktion der Delinquenz und einer Abnahme der Schulden, der Zunahme von Kontakten außerhalb der Drogenszene und an der Verbesserung der Arbeitsfähigkeit messen. Viele Veränderungen stellen sich aber sehr oft erst nach zwei bis mehreren Jahren Behandlungsdauer ein, nach einer Anfangsphase, geprägt durch Überlebenshilfe, Kriseninterventionen, sozialarbeiterische Unterstützung und Auseinandersetzungen um das Setting. Die vielen positiven Entwicklungen sollen jedoch nicht über die Tatsache hinwegtäuschen, dass es auch langjährige Betreuungen gibt, bei denen keine wesentlichen Veränderungen zu beobachten sind, die Betroffenen aber im Rahmen der „harm reduction“ vor dem weiteren Zerfall und vor dem Tod bewahrt werden können.
Die Letalität unter heroingestützter Behandlung in der Schweiz liegt wesentlich tiefer (ca. 1\% pro Jahr) als unter methadongestützter Behandlung (ca. 2,5\% pro Jahr); beide Behandlungsarten reduzieren die Sterblichkeit gegenüber nicht behandelten Drogenabhängigen (5-10\% pro Jahr) äußerst stark. AIDS-assoziierte Todesfälle standen mit 39\% im Vordergrund, gefolgt von anderen somatischen Krankheiten (16\%), Suiziden (11\%), Unfällen (8\%) und Intoxikationen (6\%; nicht im Rahmen der Substitution).

\section{Derzeitige Problemstellungen}

Da Heroin oft in Kombination mit Kokain als Cocktail eingenommen wird, stellt der Kokain-Beikonsum in den Projekten ein großes Problem dar. Fundierte Erfahrungen mit Kokainsubstitution konnten nach einem kurzen Versuch, der vorwiegend aus politischen Gründen abgebrochen wurde, nicht gemacht werden. In der Schweiz, wie in den meisten Ländern Europas, geht der Trend in der Drogenszene deutlich weg vom intravenösen Konsum zu rauchbaren Praktiken und Sniffen. Unter der oralen Heroinsubstitution fehlt aber vielen dieser sehr schwer abhängigen und marginalisierten Patient(inn)en das Gefühl des „flash“, was sie oft mit Kokaineinnahme zu kompensieren suchen. Eine rauchbare Heroinform zur Substitution würde den Kokainkonsum bei etlichen Patient(inn)en einschränken helfen. Weitere limitierende Faktoren in der Behandlung stellen die strengen Auflagen an die Behandlung dar (Gesprächsfrequenz, Mitgabebestimmungen etc.), welche es einer beträchtlichen Gruppe von stark drogenabhängigen Menschen nicht möglich machen, an einer derart stark strukturierten Behandlung teilzunehmen. Aus diesen Gründen sind in vielen Heroinprojekten in der Schweiz Behandlungsplätze frei, obwohl eine größere Gruppe, mit gescheiterten vorgängigen Behandlungsversuchen, von einer heroingestützten Therapie profitieren könnte.

\section{Dr. med. Daniel Meili}

ARUD Zürich, Poliklinik Zokl2

Konradstrasse 1

8005 Zürich

Schweiz

E-mail: arud@access.ch 